CLINICAL STUDY

\title{
Cardiovascular risk factors and arterial rigidity are similar in asymptomatic normocalcemic and hypercalcemic primary hyperparathyroidism
}

Karen M Tordjman, Marianna Yaron, Elena Izkhakov, Etty Osher, Galina Shenkerman, Yonit Marcus-Perlman and Naftali Stern

The Sackler Faculty of Medicine, Institute of Endocrinology, Metabolism and Hypertension, Tel Aviv Sourasky Medical Center, Tel Aviv University, 6 Weizmann Street, Tel Aviv 64239, Israel

(Correspondence should be addressed to K M Tordjman; Email: karentor@tasmc.health.gov.il)

\begin{abstract}
Objective: It is still uncertain whether mild primary hyperparathyroidism (PHPT) carries the same risk for increased cardiovascular (CV) morbidity as the more severe symptomatic form. In recent years, the even more subtle normocalcemic (NC) variant is being increasingly recognized. We sought to compare the prevalence of $\mathrm{CV}$ risk factors in patients with $\mathrm{NC}$ - and hypercalcemic (HC)-PHPT, and to examine whether they differ on a battery of non-invasive vascular parameters.

Design/subjects/methods: A retrospective study of two cohorts of patients with PHPT in a referral center: 32 subjects with NC-PHPT and 81 subjects with HC-PHPT, compared for the presence of clinical and biochemical risk factors, and CV morbidity. Non-invasive parameters of arterial stiffness (augmentation index; pulse wave velocity; and vascular compliance indices, $\mathrm{C} 1$ and $\mathrm{C} 2$ ) were extracted from the data of gender- and age-matched subsets of these patients, and were related to those of a group of matched control subjects.

Results: Despite a similar prevalence of hypertension $(\sim 62 \%)$, hyperlipidemia $(\sim 30 \%)$, and impaired glucose metabolism in both PHPT groups, CV or cerebrovascular disease was more common in the HC-PHPT group (24.7 vs $3.1 \%, P=0.007)$. Arterial stiffness parameters did not differ in the three groups, and were unrelated to serum calcium or parathyroid hormone concentration.

Conclusions: NC-PHPT and HC-PHPT subjects exhibit similar high rates of traditional CV risk factors, and have comparable indices of arterial stiffness. The lower clinical CV morbidity observed with NC-PHPT remains unexplained, and requires confirmation. Until then, the CV risk associated with NC-PHPT should not be underestimated.
\end{abstract}

European Journal of Endocrinology 162 925-933

\section{Introduction}

Asymptomatic primary hyperparathyroidism (PHPT) is said to be the third most common endocrine diagnosis, with an approximate annual incidence of 20/100 000 (1). However, the most cost-effective way of treating mild, or the so-called, asymptomatic disease is still debated, and indications for surgery continue to evolve (2).

Metabolic abnormalities that are conducive to cardiovascular (CV) morbidity are often seen in PHPT, and are felt to be more common than in the general population. While not entirely supported by the American data, excess CV mortality has been consistently reported among Scandinavian patients with PHPT (3-6). It has been argued that the excess mortality seen among patients with PHPT belongs to the era of severe symptomatic disease, whereas nowadays the disease is mostly recognized in a mild asymptomatic state characterized by lower serum calcium concentrations
$(2,7)$. Although there is some suggestion that the degree of hypercalcemia is directly correlated with the increased CV risk (8), an atherogenic metabolic profile has been reported in patients with normocalcemic (NC)PHPT (9). The issue is further compounded by the lack of agreement in the literature over the possible amelioration of metabolic parameters, or the putative reversibility of hypertension and myocardial hypertrophy following successful surgery $(2,10)$. Although surgery has been advocated for all patients with asymptomatic or mild PHPT as a means of reducing CV morbidity (11), reliable current prospective data regarding the $\mathrm{CV}$ risk of patients with mild asymptomatic PHPT are lacking. Therefore, the role of surgery in this setting is debatable. In a 2-year follow-up, an ongoing Scandinavian study recently reported no improvement in CV risk factors among patients with mild PHPT randomly assigned to surgery compared with patients randomized to observation only (12). 
We recently reported the clinical characteristics of asymptomatic NC-PHPT (13). As this is a fairly recently recognized entity that has emerged mostly as a result of bone density-screening procedures, our original report had focused on bone metabolism parameters and parathyroid pathology. However, we did report a high prevalence of metabolic abnormalities and hypertension among this elderly group of patients, but only one case of myocardial infarction over a follow-up period that lasted as long as 13 years.

The general aim of the present study was to further characterize the associated CV risk factors in these patients, and to compare them with those found in ageand gender-matched patients with mild hypercalcemic (HC)-PHPT. An additional specific goal was to analyze vascular function parameters to try and determine whether subjects with NC-PHPT differ from those with mild HC-PHPT, and/or from control subjects matched for age, gender, and the presence of $\mathrm{CV}$ risk factors.

\section{Methods}

\section{Study design and patients}

This was a retrospective, case-control study of unselected consecutive patients seen for a diagnosis of PHPT (ICD-9-CM code 252.01) at the Institute of Endocrinology at Tel Aviv, Sourasky Medical Center between 1998 and 2003. The original communication reported on a cohort of 32 patients with bona fide NC-PHPT, after potential causes of secondary hyperparathyroidism were actively excluded. Namely, vitamin D deficiency when present was actively corrected, deficient calcium intake was supplemented, and on the occurrence of significant hypercalciuria, a thiazide diuretic was administered. The subjects included are those in whom parathyroid hormone (PTH) levels remained elevated despite all these maneuvers (13). In the current study, we also included 81 consecutive patients with mild HC-PHPT, who were seen in the department during the same period.

All relevant clinical and biochemical data were retrieved from the patients' charts in both entire cohorts. In addition, data from non-invasive vascular studies (methodology detailed below), available on a subset of subjects in both groups, were also retrieved, and compared to age- and gender-matched control subjects in whom a diagnosis of PHPT could be excluded. Such studies are offered to patients felt to carry diagnoses conveying a CV risk. As this is a retrospective study, subjects were not required to provide written informed consent, but the study received approval from the institutional ethical review committee.

\section{Criteria for the diagnosis of associated metabolic abnormalities}

A diagnosis of hypertension was made either if a subject received antihypertensive medications or if the blood pressure in the clinic equaled or exceeded $140 / 90 \mathrm{mmHg}$ on at least two occasions (14). Hyperlipidemia was diagnosed as total cholesterol equal to or higher than $200 \mathrm{mg} / \mathrm{dl}$ or/and triglycerides equal to or higher than $150 \mathrm{mg} / \mathrm{dl}$ after a 12 -h fast, or if the patient was being medicated for such a condition. Likewise, a diagnosis of diabetes was made using the current criteria (15), or in patients treated with hypoglycemic drugs. Impaired fasting glucose was considered if two separate fasting glucose levels were above $100 \mathrm{mg} / \mathrm{dl}$. A diagnosis of CV disease was either self-reported, or taken from a diagnosis appearing on a referral document. In some cases, a diagnosis was further substantiated by ancillary studies available for review such as discharge summaries, heart catheterization reports, or results of Doppler ultrasonographic studies of carotid or peripheral arteries.

\section{Biochemical and hormonal determinations}

All biochemical analytes were determined on a Hitachi 747 random-access analyzer or a Bayer Advia 1650 analyzer with identical analytical performances. The normal range for serum calcium was $8.5-10.5 \mathrm{mg} / \mathrm{dl}$. Intact PTH (iPTH) was measured in cold plasma with a solid-phase, two-site chemiluminescent enzyme-labeled immunometric assay (immulite iPTH, Diagnostic Products Corporation, Los Angeles, CA, USA) with a reference range of $12-72 \mathrm{pg} / \mathrm{ml}$. The serum concentration of $25(\mathrm{OH})$-vitamin $\mathrm{D}$ was determined in serum with an in-house competitive protein-binding radioassay after lipid extraction preparative sephadex LH-20 chromatography (16). Using this assay, which served for many years the central national vitamin laboratory, the results were stratified according to nationally validated norms that differ somewhat from the currently used references. Indeed, normal values for a young (mean age 41 years) and active Israeli population that included $\sim 50 \%$ of outdoor workers ranged from 23.4 $\pm 8.6 \mathrm{ng} / \mathrm{ml}$ in winter to $28.0 \pm 8.7 \mathrm{ng} / \mathrm{ml}$ in summer (17). By this assay, vitamin D sufficiency was considered for concentrations in excess of $20 \mathrm{ng} / \mathrm{ml}$, insufficiency lay between 15 and $20 \mathrm{ng} / \mathrm{ml}$, whereas deficiency was any value below $15 \mathrm{ng} / \mathrm{ml}$ (18). Serum concentrations of $1,25(\mathrm{OH})_{2}$-vitamin D were determined by a RIA (Dia Sorin, Stillwater, MN, USA); a normal value for this assay lies between 10 and $65 \mathrm{pg} / \mathrm{ml}$.

\section{Non-invasive vascular studies}

Subjects were examined in the fasting state (at least $3 \mathrm{~h}$ before the examination). Examinees were requested to abstain from consuming caffeinated beverages on the day of the measurements. Subjects rested in silence in a supine position for at least $10 \mathrm{~min}$ in a quiet, temperature-controlled room. 
All the studied patients underwent a battery of noninvasive assessments of arterial stiffness with a number of commercially available devices according to the recommended procedures (19). The following were used for the assessments:

(i) The SphygmoCor System (AtCor Medical, Sydney, Australia). Pulse wave analysis (SphygmoCor) generates the augmentation index (AI), which is an indirect measure of arterial stiffness $(20,21)$.

Peripheral pressure waveforms were recorded from the radial artery at the wrist using applanation tonometry with a high-fidelity micromanometer (SPC-301; Millar Instruments, Houston, TX, USA). The AI derived from this technique was defined as the augmentation pressure divided by the pulse pressure, and was expressed as a percentage. As this index is affected by heart rate, the results were normalized for a heart rate of 75 beats/min.

(ii) The Complior device (Colson, Paris, France). Pulse wave velocity (PWV), a gold standard of large arterial stiffness measurement, is measured between the carotid and the femoral artery (Complior) $(22,23)$. PWV was obtained transcutaneously over the right common carotid artery and the right femoral artery, and the transit time (T-time) was determined from the time delay $(t)$ between the appearances of two feet of the corresponding waveforms. PWV was calculated from the measurements of the pulse transit time and the distance traveled by the pulse between two recording sites as $\mathrm{PWV}=\mathrm{D}$ (meters)/transit time (seconds). The distance between the recording sites was measured with a tape over the surface of the body.

(iii) Radial artery pulse wave analysis using CR-2000 (Hypertension Diagnostics, Eagan, MN, USA). Applanation tonometry (HDI, CR-2000) generates parameters of large (C1) and small (C2) artery compliance $(24,25)$. The radial artery waveform was obtained with a sensor positioned over the artery, and was calibrated using an oscillometric method on the opposite arm. A beat-marking algorithm determined the beginning of systole, peak systole, onset of diastole, and end diastole for all beats in a 30-s measurement period. An average beat determination was constructed, and a parameter-estimating algorithm was applied to define a third-order equation that replicated the diastolic decay and waveform. Small artery elasticity or oscillatory components $(\mathrm{C} 2 \mathrm{ml} / \mathrm{mmHg} \times 100)$ and large artery elasticity or capacitive components (C1 $\mathrm{ml} / \mathrm{mmHg} \times 10)$ were then determined based on the modified Windkessel model. Blood pressure measurements were obtained on a validated, automated blood pressure monitor (Omron Healthcare, Bannockburn, IL, USA), with the radial artery kept at heart level during the measurement.

\section{Statistical analysis}

Data are expressed as mean \pm s.D. Student's $t$-test or non-parametric test (the Mann-Whitney test) was used to compare continuous variables in the groups of patients with PHPT. Comparison of frequency of co-morbidities was carried out using Fisher's exact test or Pearson's $\chi^{2}$-test. Results of vascular studies were compared among the three groups by one-way ANOVA using Bonferroni's post-test analysis. ANOVA with the Kruskal-Wallis method with Dunn's post-test was used when non-parametric methods were indicated. Correlations between parameters were sought by performing Pearson's linear regressions or Spearman's statistics for non-parametric data, while stepwise multiple linear regressions were carried out to examine the effect of several parameters on any of the measures. All computations were performed with the SPSS version 17 statistical software (SPSS Inc., Chicago, IL, USA). Significance was considered for a $P$ value $<0.05$.

\section{Results}

\section{Hyperparathyroid patients' demographic, clinical, and biochemical data}

Data of NC- and HC-PHPT patients at the time of presentation in our clinic are presented in Table 1. Groups did not differ with respect to age or gender distribution.

Although iPTH levels were similar in both groups, by definition, the two groups differed significantly with regard to their serum calcium concentrations. This was also associated with a significantly higher urinary calcium excretion in the HC group (311 \pm 144 vs 242 $\pm 139 \mathrm{mg} / 24 \mathrm{~h}, P=0.003)$. The proportion of patients with significant hypercalciuria $(>300 \mathrm{mg} / 24 \mathrm{~h})$ at presentation was significantly higher in the HC group (46\%) than in the NC group $(19 \%, P=0.008)$. The two groups did not differ in their $25(\mathrm{OH})$-vitamin D levels. If anything, there was a trend for a greater prevalence of vitamin D deficiency among patients with HC-PHPT (39\% of those in whom it was measured). Nonetheless, vitamin supplementation was far more frequent among the NC-PHPT subjects, and so was calcium administration, in part a reflection of the attempts to lower PTH in these patients. Although this was not significant, there was clearly a trend for a more liberal use of thiazide diuretics in the NC patients in whom the risk of worsening hypercalcemia was not a concern (Table 2).

Renal function assessed by creatinine clearance was preserved in the NC group, confirming the notion that the elevated iPTH was not a reflection of impaired kidney function in this group.

Follow-up duration following the diagnosis of PHPT was similar in both groups: $3.7 \pm 2.7$ years in $\mathrm{NC}$ and $4.5 \pm 3.0$ years in $\mathrm{HC}, P=\mathrm{NS}$. Not all NC-PHPT subjects 
Table 1 Demographic and biochemical data of normocalcemic-primary hyperparathyroidism (NC-PHPT) and hypercalcemic (HC)-PHPT patients at the time of the initial evaluation.

\begin{tabular}{|c|c|c|c|}
\hline Variable & NC-PHPT mean (S.D.) & HC-PHPT mean (S.D.) & $\boldsymbol{P}$ \\
\hline$n$ & 32 & 81 & \\
\hline Gender (F/M) & $27 / 5$ & $56 / 25$ & NS \\
\hline Age (years) & $60.5(10.5)$ & $62.5(13)$ & NS \\
\hline Serum calcium $(\mathrm{mg} / \mathrm{dl})$ & $9.8(0.46)$ & $11.5(1.2)$ & $<0.0001$ \\
\hline Corrected calcium (mg/dl) $)^{a}$ & $9.6(0.45)$ & $11.2(1.3)$ & $<0.0001$ \\
\hline Serum phosphorus (mg/dl) & $2.9(0.47)$ & $2.7(0.5)$ & NS \\
\hline iPTH $(\mathrm{pg} / \mathrm{ml})$ & $123(56)$ & $174(188)$ & NS \\
\hline Urinary calcium excretion (mg/24 h) & $242(139)$ & $311(144)$ & 0.003 \\
\hline Creatinine clearance $(\mathrm{ml} / \mathrm{min})^{b}$ & $99(21.6)$ & $86(29)$ & NS \\
\hline Serum 25(OH)-vitamin D $(\mathrm{ng} / \mathrm{ml})^{\mathrm{c}}$ & $22.7(7.8)$ & $20.7(10.5)$ & NS \\
\hline Serum $1,25(\mathrm{OH})_{2}$-vitamin $D(\mathrm{pg} / \mathrm{ml})$ & $47.4(25.7)$ & $55.2(17.4)$ & NS \\
\hline
\end{tabular}

alonized calcium was infrequently measured in both groups, and thus, corrected serum calcium was used as an indirect assessment of ionized calcium. This was calculated in all patients by adding or subtracting $0.8 \mathrm{mg} / \mathrm{dl}$ of calcium for each $1.0 \mathrm{mg} / \mathrm{dl}$ of serum albumin above or below 4.0 .

${ }^{\mathrm{b}}$ Creatinine clearance rate was calculated from a $24-\mathrm{h}$ urine collection.

${ }^{c}$ Serum 25(OH)-vitamin D levels were available for 27 of the normocalcemic subjects, but only for 18 of the hypercalcemic patients. Note (in Methods) that reference data for vitamin D levels are different from the current levels, and are a result of the in-house assay employed.

included in the original study have since remained under our care. However, of those who did, none went on to develop hypercalcemia later on.

\section{Associated metabolic and cardiovascular diagnoses}

The prevalence of hypertension was high and identical in both groups. Indeed, two-thirds of all PHPT patients suffered from this condition. In addition to the strong association with hypertension, often viewed as a manifestation of the disease itself, diabetes or impaired fasting glucose and dyslipidemia have also been reported to be over-represented in PHPT. It is notable that although the rates of hypertension, glucose homeostasis abnormalities, and hyperlipidemia were identical in both groups (Table 2), clinically manifest ischemic heart disease was significantly more prevalent in the HC cohort. When the frequencies of cerebrovascular and ischemic heart disease, both manifestations of vascular disease, were combined, the excess of CV morbidity in the HC group became highly significant $(24.7$ vs $3.1 \%, P=0.007)$. It should be pointed out that none of the patients with a history of $\mathrm{CV}$ disease was referred for endocrine management close to the $\mathrm{CV}$ event or in relation to it. Finally, the

Table 2 Comparison of the frequency of metabolic and cardiovascular abnormalities accompanying a diagnosis of primary hyperparathyroidism (PHPT), and of the pattern of use of medications likely to affect calcium metabolism in both groups of subjects.

\begin{tabular}{|c|c|c|c|}
\hline Condition & Normocalcemic $n(\%)$ & Hypercalcemic $n(\%)$ & $\boldsymbol{P}$ \\
\hline Hypertension & $20(62.5)$ & $50(61.7)$ & NS \\
\hline Hyperlipidemia & $10(31.3)$ & $22(27.2)$ & NS \\
\hline \multicolumn{4}{|l|}{ Thyroid dysfunction } \\
\hline Hypothyroid & $4(12.5)$ & $7(8.6)$ & NS \\
\hline Hyperthyroid & $1(3.1)$ & $3(3.7)$ & NS \\
\hline Diabetes or impaired fasting glucose & $6(19)$ & $26(32.1)$ & NS \\
\hline CVA $^{\mathrm{a}}$ & 0 & $4(4.9)$ & NS \\
\hline$I H D^{b}$ & $1(3.1)$ & $16(19.8)$ & 0.038 \\
\hline IHD and/or CVA & $1(3.1)$ & $20(24.7)^{\mathrm{c}}$ & 0.007 \\
\hline Cardiac dysrhythmia & $1(3.1)$ & $4(4.9)$ & NS \\
\hline \multicolumn{4}{|l|}{ Medications } \\
\hline Thiazide diuretics & $5(15.6)$ & $8(9.9)$ & NS \\
\hline Loop diuretics & 0 & $6(7.4)$ & NS \\
\hline ACEi or/and ARB ${ }^{d}$ & $12(37.5)$ & $29(35.8)$ & NS \\
\hline Calcium channel blockers & $12(37.5)$ & $32(39.5)$ & NS \\
\hline$\beta$-Blockers & $5(15.6)$ & $20(24.7)$ & NS \\
\hline Calcium supplements & $11(34.4)$ & $1(1.2)$ & $<0.0001$ \\
\hline Vitamin D supplements & $14(43.8)$ & $3(3.7)$ & $<0.0001$ \\
\hline
\end{tabular}

Note that patients received at times more than one medication for the treatment of hypertension or ischemic heart disease. Not detailed in the table, a few patients received $\alpha$-blockers and spironolactone.

${ }^{a}$ CVA, cerebrovascular accident.

${ }^{\mathrm{b}} \mathrm{IHD}$, ischemic heart disease.

In the HC cohort, two patients had both IHD and CVA.

${ }^{\mathrm{d}} \mathrm{ACEi}$, angiotensin-converting enzyme inhibitors; ARB, angiotensin receptor blockers. 
Table 3 Demographic, anthropometric, selected biochemical data, and associated cardiovascular risk factors in patients with normocalcemic-primary hyperparathyroidism (NC-PHPT) and hypercalcemic (HC)-PHPT and a group of non-PHPT control subjects selected to match the patients' population. Data are expressed as mean \pm s.D. For categorical variables, the absolute number is given with the rate as percentage in parentheses.

\begin{tabular}{|c|c|c|c|c|}
\hline & NC-PHPT & HC-PHPT & Control & $\boldsymbol{P}$ \\
\hline$n(\mathrm{~F} / \mathrm{M})$ & $13(11 / 2)$ & $12(9 / 3)$ & $25(20 / 5)$ & NS \\
\hline Age (years) & $65.2 \pm 7.6$ & $67.8 \pm 10.5$ & $65.9 \pm 9.3$ & NS \\
\hline BMI $\left(\mathrm{kg} / \mathrm{m}^{2}\right)$ & $27.5 \pm 5.2$ & $26.9 \pm 4.0$ & $27.2 \pm 5.7$ & NS \\
\hline System BP (mmHg) & $136.3 \pm 19.6$ & $142.3 \pm 25.0$ & $137.4 \pm 18.3$ & NS \\
\hline Diastolic BP (mmHg) & $74.3 \pm 7.3$ & $76.5 \pm 9.1$ & $72.1 \pm 8.4$ & NS \\
\hline Serum calcium (mg/dl) & $9.6 \pm 0.4$ & $11.1 \pm 0.3$ & $9.2 \pm 0.4$ & $<0.0001$ \\
\hline iPTH $(\mathrm{ng} / \mathrm{ml})$ & $103.3 \pm 25.5$ & $158.6 \pm 113.6$ & $20.1 \pm 3.9$ & 0.02 \\
\hline Hypertension & $9(6 \overline{9} .2)$ & $9(\overline{75})$ & $17(\overline{68})$ & NS \\
\hline Diabetes or IFG & $2(15.4)$ & $5(41.7)$ & $9(36)$ & NS \\
\hline Hyperlipidemia & $8(61.5)$ & $8(66.7)$ & $17(68)$ & NS \\
\hline Cardiovascular disease history & $0(0)$ & $3(25)$ & $3(12)$ & $0.048^{a}$ \\
\hline
\end{tabular}

${ }^{\mathrm{a} B y}$ Pearson's $\chi^{2}$-test.

co-occurrence of a metabolically relevant thyroid dysfunction could have affected the prevalence of overt CV disease; however, both hypothyroidism and hyperthyroidism were equally distributed in both groups.

\section{Non-invasive vascular function assessment}

For these studies, we retrieved the data of $13 \mathrm{NC}$ - and 12 HC-PHPT patients. In addition, out of our database of vascular studies conducted during the same period and by the same operator (MY), we selected a group of 25 control subjects matched for age, gender, and the presence of $\mathrm{CV}$ risk factors (Table 3). None of the subjects currently smoked, and all had normal thyroid function. All three groups were also matched for medications. With the exception of thiazide diuretic use which was exceptional in the HC-PHPT group (one subject only), the use of all other medications affecting the CV system was similar in all three groups. Notably, $\beta$-blockers were used by $33 \%$ of the HC-PHPT, $31 \%$ of the NC-PHPT, and $36 \%$ of the control subjects; calcium channel blockers were used by 33, 46, and $36 \%$ respectively. Likewise, statins were widely prescribed as $54 \%$ of the NC-PHPT, 58\% of the HC-PHPT, and $64 \%$ of the control subjects were taking them at the time of the study.

As these studies are not covered by insurers, the subjects who offered to undergo them were probably chosen because they were felt to have higher than average CV risk factors. As shown, both groups of patients and controls were well matched for the presence of risk factors. Likewise, the subgroups of PHPT subjects on whom vascular studies were performed were, by and large, representative of the entire cohort. The only significant difference was for the prevalence of hyperlipidemia among the HC-PHPT subjects, which was present in a little below a third of the entire HC cohort, but in two-thirds of the $\mathrm{HC}$ subjects who underwent the vascular assessment $(P<0.001)$. In these subgroups too, despite a similar prevalence of $\mathrm{CV}$ risk factors, the patients with HC-PHPT had a higher rate of CV diseases, while none of the NC patients carried such a diagnosis $(P=0.048)$.

Given the apparent difference in CV morbidity between the PHPT groups, we hypothesized that hypercalcemia per se could play a role by affecting arterial stiffness and vascular plasticity. Our measurements included two parameters of arterial stiffness, the PWV and the AI. These were identical among the two PHPT subgroups and the control subjects (Fig. 1). Interestingly, despite the presence of multiple vascular risk factors, the mean values for these parameters were relatively normal in all three groups. Likewise, the proportion of subjects with abnormal values (PWV $>10 \mathrm{~m} / \mathrm{s}$ or $\mathrm{AI}>40 \%$ ) was equal in all three groups. The two indices of vessel compliance were also not different between the groups (Fig. 2). C1, the large vessel elasticity index, was on average normal $(>13.7 \mathrm{ml} / \mathrm{mmHg} \times 10)$. However, $\mathrm{C} 2$, the index of small artery elasticity, was low in all three groups, attesting to the presence of vascular bed compliance impairment (normal $>5.5 \mathrm{ml} / \mathrm{mmHg} \times 100$ ) in these subject groups.

By analyzing the entire database of vascular studies, we sought to identify correlations that might shed light on variables likely to be involved in the protection from CV morbidity seen in NC-PHPT subjects. However, the only correlations we found were, as expected, between the indices of arterial stiffness AI and PWV $(r=0.44$, $P=0.019$ ), and between the indices of arterial compliance $\mathrm{C} 1$ and $\mathrm{C} 2 \quad(r=0.643, P<0.0001)$. By univariate analysis, we found the known inverse correlation between systolic blood pressure and $\mathrm{C} 1$ and $\mathrm{C} 2$, and a positive correlation with PWV and AI. All these indices were also significantly affected by increasing age. However, neither body mass index nor serum levels of calcium, phosphorus, or PTH were 

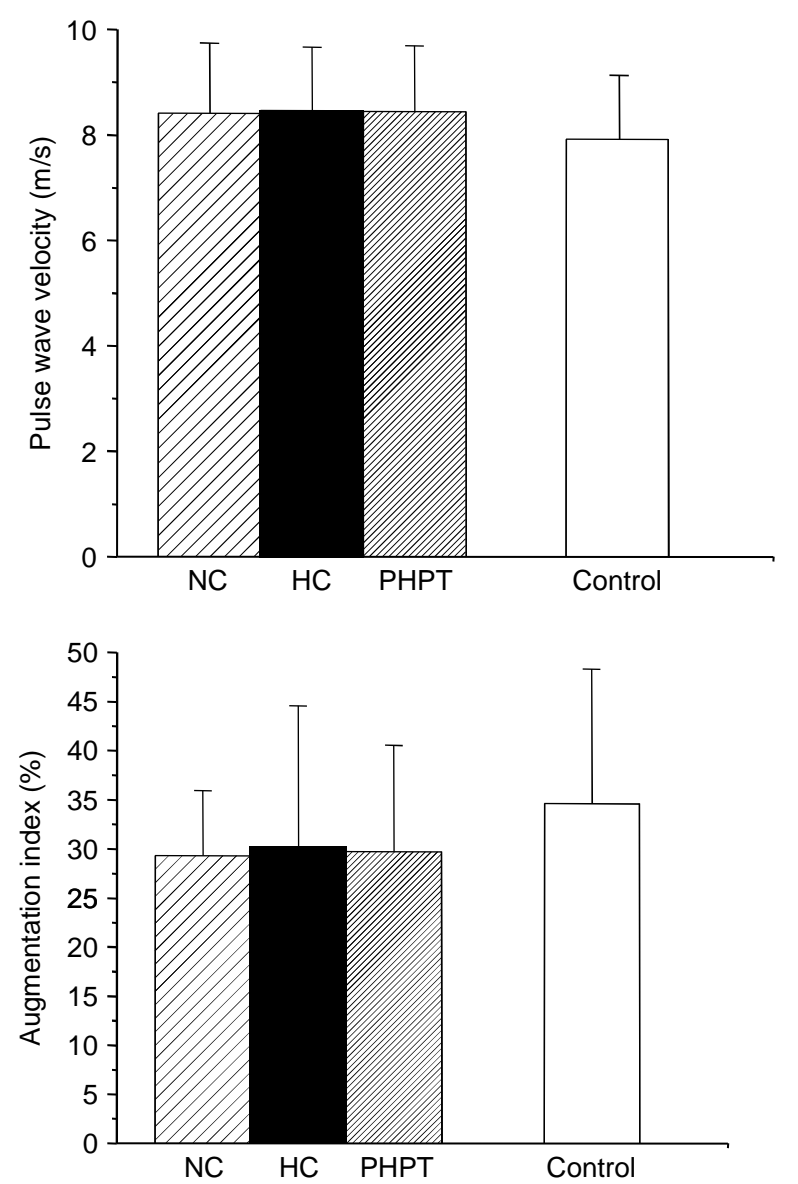

Figure 1 Arterial stiffness assessment as reflected by the pulse wave velocity (top) and the augmentation index (bottom) obtained by waveform analysis. None of these measures differed among the three groups: NC-PHPT $(n=13)$, HC-PHPT $(n=12)$, or controls $(n=25)$. The combined PHPT groups is also depicted. Values are given as mean \pm s.D. Normal values are given in the results.

shown to affect any of the variables. Likewise, a stepwise multiple regression analysis and selection of the best subset in a model that adjusted for systolic blood pressure, age, gender, and the presence of diabetes failed to reveal any differences between the groups.

\section{Discussion}

In the present study, we first completed the metabolic characterization of a fairly newly recognized entity, NC-PHPT. We now show that with the exception of serum calcium and the degree of urinary calcium excretion, which are inherently lower in this group, both subsets of PHPT patients are essentially identical, particularly with respect to the presence of traditional $\mathrm{CV}$ risk factors. Given the patients' sample size, this study was not powered to confirm the alleged increased prevalence of metabolic risk factors in PHPT. However, the elevated rates of hypertension, dyslipidemia, and impaired glucose metabolism found in all PHPT subjects appear to exceed those found in the general age-matched Israeli population, but are in keeping with those found by Luboshitzky et al. in a recent study of PHPT subjects conducted in Israel (26, $27)$. Given these risk factors, the prevalence of clinical CV manifestations in the HC-PHPT subjects is of no surprise. The lack of such in the NC-PHPT subjects was unexpected, particularly in view of the similar known duration of PHPT since diagnosis in both groups. Although this difference is intriguing, one cannot exclude it was a chance finding stemming from the limited sample size.

In order to get some insight into some preclinical vascular characteristics that might differentiate the two
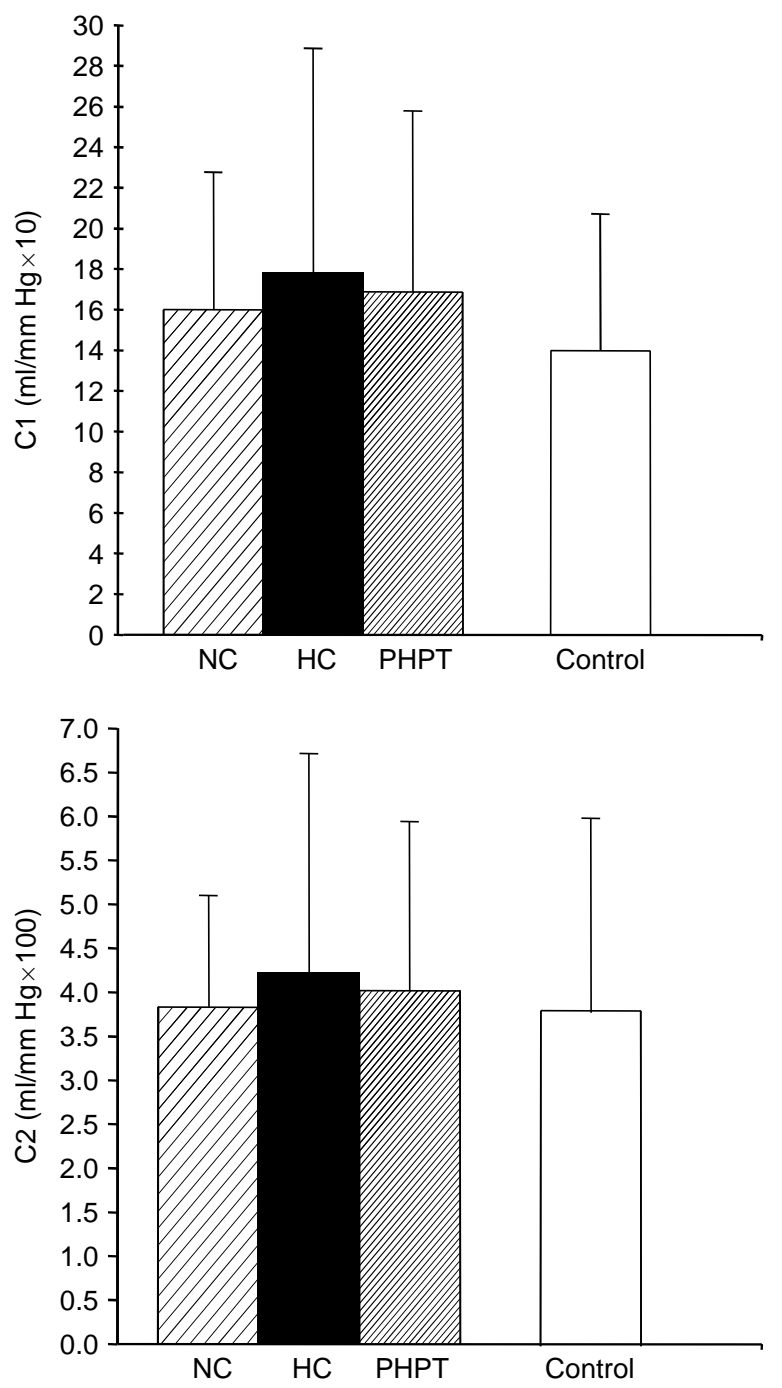

Figure 2 Capacitive, large artery compliance C1 (top), and oscillatory, small artery compliance $\mathrm{C} 2$ (bottom) derived from the application of the Windkessel model of the pulse contour analysis obtained by applanation tonometry. Values are given as mean \pm S.D. No difference was observed in any of these for the three groups. 
PHPT groups, we examined a number of vascular function measurements obtained on subsets of PHPT patients and on a group of control subjects adequately matched for age, gender, and the presence of traditional CV risk factors. We found no tangible difference in any of the variables studied among the groups of subjects. With the exception of $\mathrm{C} 2$, a measure of small artery compliance, the parameters studied were not strikingly abnormal. Although NC-PHPT subjects were overrepresented in these studies (13/32 vs $12 / 81$ in the HC-PHPT group), the fact that their data were not more favorable than those of the HC-PHPT subjects argues against a sampling bias.

Preclinical abnormal vascular characteristics have been reported in patients with PHPT. An elevated AI that reflects increased arterial stiffness and correlates with CV risk (28) has been described in patients with mild PHPT (29). In this report by Smith et al. AI was significantly higher in subjects with PHPT than in controls (36 vs $25 \%$ ), and in a multiple regression analysis, these authors found serum calcium to be its strongest predictor. In a different study, Rubin et al. also reported a somewhat higher AI in PHPT subjects than in controls (28 vs 25\%); however, although PHPT itself was an independent predictor of AI, like in our present study, serum calcium itself was not (30). In yet another report, Barletta et al. showed that PWV, also a measure of arterial stiffness, was no different in subjects with mild PHPT than in age- and sex-matched controls. In this paper, subjects were very similar to those in our current study, and their PWV was also almost identical to that we observed. Significantly, it was identical in patients and controls $(8.8 \pm 1.6$ vs $8.2 \pm 3.5 \mathrm{~m} / \mathrm{s}$ ), and was likewise unaffected by surgery when studied again 6 months after correction of the metabolic abnormality (31).

Decreased arterial compliance indices $\mathrm{C} 1$ and $\mathrm{C} 2$ derived from diastolic pulse contour analysis have also been associated with conditions that portend increased $\mathrm{CV}$ risk such as hypertension, smoking, and diabetes. The small artery compliance index $\mathrm{C} 2$, in particular, has been reported to be a sensitive marker of early vascular disease (32). To our knowledge, these parameters have not been reported previously in subjects with PHPT. It is noteworthy that although no difference was noted between the groups studied, all three groups had reduced $\mathrm{C} 2$ as a reflection of the high rate of risk factors these subjects displayed.

Carotid intima-media thickness (IMT) was not routinely measured in our laboratory at the time these studies were conducted. Increased IMT, viewed as an early sign of atherosclerosis, has been inconsistently described in patients with PHPT (33-37), and it is unclear whether it is present in the absence of traditional risk factors. Furthermore, it appears not to be associated with the degree of hypercalcemia, and to be unaffected by parathyroid surgery $(34,36)$.
A potential limitation of this study is that endothelial function was not investigated. It has been suggested that the endothelium, a recognized target organ for PTH, is the site through which chronically elevated PTH can induce hypertension, possibly by affecting the nitric oxide synthase system (38). Relevantly, endothelial vasodilatory dysfunction, considered as an early event underlying atherosclerosis, has been documented in hyperparathyroidism. It has been suggested that endothelial dysfunction can be reversed by successful surgery (39-41). However, it should be stressed that these studies could not distinguish between the effect of restoration of normocalcemia and that of the return of PTH to normal as the immediate reason for the improvement. In this respect, it is noteworthy that acute calcium infusion in normal volunteers can emulate the vasodilatory disturbances seen in PHPT, suggesting a direct role for calcium itself (42). As our two cohorts of patients had similar concentrations of $\mathrm{PTH}$, comparing their respective endothelial function parameters and relating them to serum calcium concentrations would have been a valuable adjunct to this study.

Finally, another obvious limitation of this study is its retrospective nature. Although arterial stiffness measurements were available only on subsets of patients, these subjects appeared to adequately represent their respective cohorts. As we anticipated to detect some of the abnormalities described in the literature, the fact that with the exception of $\mathrm{C} 2$, most of the measures were not grossly abnormal came somewhat as a surprise. This could generally be seen as evidence to the adequate medical treatment patients received. At any rate, the fact that similar arterial stiffness parameter results were obtained in all three groups is a strong argument against a sampling bias, and supports the idea that serum calcium level in itself is not a major determinant of any of them.

In summary, despite exhibiting the typical CV risk factors found in HC-PHPT, our cohort of NC-PHPT patients seemed to enjoy a relative protection from $\mathrm{CV}$ morbidity. This was not explained by the non-invasive arterial stiffness parameters examined in this study, as these were identical to those of HC-PHPT patients and those of normal controls, and were not correlated with serum calcium. A study that would assess the respective endothelial function of these subjects is needed. Finally, until prospective data are available on the natural cardiometabolic course in subjects with NC-PHPT, we believe that the low prevalence of $\mathrm{CV}$ disease found in the present study should not affect the therapeutic choices taken in this newly recognized condition.

\section{Declaration of interest}

The authors declare that there is no conflict of interest that could be perceived as prejudicing the impartiality of the research reported. 


\section{Funding}

This research did not receive any specific grant from any funding agency in the public, commercial, or non-for-profit sector.

\section{Acknowledgements}

The authors wish to acknowledge Mr Doron Comaheshter and Ms Esther Shabtai for their skillful statistical assistance.

\section{References}

1 Melton LJ III. The epidemiology of primary hyperparathyroidism in North America. Journal of Bone and Mineral Research 200217 (Suppl 2) N12-N17.

2 Silverberg SJ, Lewiecki EM, Mosekilde L, Peacock M \& Rubin MR. Presentation of asymptomatic primary hyperparathyroidism: proceedings of the third international workshop. Journal of Clinical Endocrinology and Metabolism 200994 351-365.

3 Hedbäck G, Tisell LE, Bengtsson BA, Hedman I \& Oden A. Premature death in patients operated on for primary hyperparathyroidism. World Journal of Surgery 1990 14 829-835.

4 Hedbäck G \& Oden A. Increased risk of death from primary hyperparathyroidism - an update. European Journal of Clinical Investigation $1998 \mathbf{2 8} 271-276$.

5 Nilsson IL, Yin L, Lundgren E, Rastad J \& Ekbom A. Clinical presentation of primary hyperparathyroidism in Europe - nationwide cohort analysis on mortality from nonmalignant causes. Journal of Bone and Mineral Research 200217 N68-N74.

6 Øgard CG, Engholm G, Almdal TP \& Vestergaard H. Increased mortality in patients hospitalized with primary hyperparathyroidism during the period 1977-1993 in Denmark. World Journal of Surgery 200428 108-111.

7 Silverberg SJ \& Bilezikian JP. Evaluation and management of primary hyperparathyroidism - author's response. Journal of Clinical Endocrinology and Metabolism 199782983.

8 Wermers RA, Khosla S, Atkinson EJ, Grant CS, Hodgson SF, O'Fallon WM \& Melton LJ III. Survival after the diagnosis of hyperparathyroidism: a population-based study. American Journal of Medicine $1998 \mathbf{1 0 4} 115-122$.

9 Hagström E, Lundgren E, Rastad J \& Hellman P. Metabolic abnormalities in patients with normocalcemic hyperparathyroidism detected at a population-based screening. European Journal of Endocrinology 2006155 33-39.

10 Heyliger A, Tangpricha V, Weber C \& Sharma J. Parathyroidectomy decreases systolic and diastolic blood pressure in hypertensive patients with primary hyperparathyroidism. Surgery $2009 \mathbf{1 4 6}$ 1042-1047.

11 Rosenbloom SJ. Evaluation and management of primary hyperparathyroidism. Journal of Clinical Endocrinology and Metabolism 199782 982-983.

12 Bollerslev J, Rosen T, Mollerup CL, Nordenström J, Baranowski M, Franco C, Pernow Y, Isaksen GA, Godang K, Ueland T, Jansson S \& on behalf of the SIPH Study Group. Effect of surgery on cardiovascular risk factors in mild primary hyperparathyroidism. Journal of Clinical Endocrinology and Metabolism $2009 \mathbf{9 4}$ 2255-2261.

13 Tordjman KM, Greenman Y, Osher E, Shenkerman G \& Stern N. Characterization of normocalcemic primary hyperparathyroidism. American Journal of Medicine 2004117 861-863.

14 Chobanian AV, Bakris GL, Black HR, Cushman WC, Green LA, Izzo JL Jr, Jones DW, Materson BJ, Oparil S, Wright JT Jr \& Roccella EJ. National Heart, Lung, and Blood Institute Joint National Committee on Prevention, Detection, Evaluation, and Treatment of High Blood Pressure; National High Blood Pressure Education Program Coordinating Committee. Journal of the American Medical Association 2003289 2560-2572.
15 Report of the Expert Committee on the Diagnosis and Classification of Diabetes Mellitus. Diabetes Care 199720 1183-1197.

16 Weisman Y, Reiter E \& Root A. Measurement of 24,25 dihydroxyvitamin D in sera of neonates and children. Journal of Pediatrics 197736 904-907.

17 Azizi E, Pavlotsky F, Vered I \& Kudish AI. Occupational exposure to solar UVB and seasonal monitoring of serum levels of 25-hydroxy vitamin D3: a case-control study. Photochemistry and Photobiology 200985 1240-1244.

18 Weisman Y. Osteoporosis and vitamin D deficiency in Israel. Public Health Reviews 200028 59-65.

19 Van Bortel LM, Duprez D, Starmans-Kool MS, Safar ME, Giannattasio C, Cockcroft J, Kaiaser DR \& Thuillez C. Clinical applications of arterial stiffness, Task Force III: recommendations for user procedures. American Journal of Hypertension 200215 445-452.

20 Chen CH, Nevo E, Fetics B, Pak PH, Yin FC, Maughan WL \& Kass DA. Estimation of central aortic pressure waveform by mathematical transformation of radial tonometry pressure: validation of generalized transfer function. Circulation 199795 $1827-1836$.

21 Pauca AL, O'Rourke MF \& Kon ND. Prospective evaluation of a method for estimating ascending aortic pressure from the radial artery pressure waveform. Hypertension 200138 932-935.

22 Asmar R, Benetos A, Topoucian J, Laurent P, Pannier B, Brisac AM, Target R \& Levy BI. Assessment of arterial distensibility by automatic pulse wave velocity measurement. Validation and clinical application studies. Hypertension 199526 485-490.

23 Laurent S, Boutouyrie P, Asmar R, Gautier I, Laloux B, Guize L, Ducimetiere P \& Benetos A. Aortic stiffness is an independent predictor of all-cause and cardiovascular mortality in hypertensive patients. Hypertension 200137 1236-1241.

24 Finkelstein SM \& Cohn JN. First- and third-order models for determining arterial compliance. Journal of Hypertension $1992 \mathbf{1 0}$ S11-S14.

25 Cohn JN, Finkelstein S, McVeigh G, Morgan D, LeMay L, Robinson J \& Mock J. Pulse wave analysis for the early detection of vascular disease. Hypertension 199526 503-508.

26 Rennert G \& Peterburg Y. Prevalence of selected chronic diseases in Israel. Israel Medical Association Journal 20013 404-408.

27 Luboshitzky R, Chertok-Shacham Y, Lavi I \& Ishay A. Cardiovascular risk factors in primary hyperparathyroidism. Journal of Endocrinological Investigation 200932 317-321.

28 Duprez DA, Kaiser DR, Whitwam W, Finkelstein S, Belalcazar A, Patterson R, Glasser S \& Cohn JN. Determinants of radial artery pulse wave analysis in asymptomatic individuals. American Journal of Hypertension 200417 647-653.

29 Smith JC, Page MD, John R, Wheeler MH, Cockcroft JR, Scanlon MF \& Davies JS. Augmentation of central arterial pressure in mild primary hyperparathyroidism. Journal of Clinical Endocrinology and Metabolism 200085 3515-3519.

30 Rubin MR, Maurer MS, McMahon DJ, Bilezekian JP \& Silverberg S. Arterial stiffness in mild primary hyperparathyroidism. Journal of Clinical Endocrinology and Metabolism 200590 3326-3330.

31 Barletta G, De Feo ML, Del Bene R, Lazzeri C, Vecchiarino S, La Villa G, Brandi ML \& Franchi F. Cardiovascular effects of parathyroid hormone: a study in healthy subjects and normotensive patients with mild primary hyperparathyroidism. Journal of Clinical Endocrinology and Metabolism 200085 1815-1821.

32 Oliver JJ \& Webb DJ. Assessment of arterial stiffness and risk of atherosclerotic events. Arteriosclerosis, Thrombosis, and Vascular Biology 200323 554-566.

33 Kosch M, Hausberg M, Vormbrock K, Kisters K, Rahn KH \& Barenbrock M. Studies on flow-mediated vasodilation and intimamedia thickness of the brachial artery in patients with primary hyperparathyroidism. American Journal of Hypertension 200013 759-764.

34 Nuzzo V, Tauchmanova L, Fonderico F, Trotta R, Fittipaldi MR, Rossi R, Trimarco B \& Lupoli G. Increased intima-media thickness of the carotid artery wall, normal blood pressure profile 
and normal left ventricular mass in subjects with primary hyperparathyroidism. European Journal of Endocrinology 2002 147 453-459.

35 Fallo F, Camporese G, Capitelli E, Andreozzi GM, Matero F \& Lumachi F. Ultrasound evaluation of carotid artery in primary hyperparathyroidism. Journal of Clinical Endocrinology and Metabolism $2003 \mathbf{8 8} 2096$-2099.

36 Kosch M, Hausberg M, Barenbrock M, Posadzy-Malaczynska A \& Kis Ran RH. Arterial distensibility and pulse wave velocity in patients with primary hyperparathyroidism before and after parathyroidectomy. Clinical Nephrology 200155 303-308.

37 Lumachi F, Ermani M, Frego M, Pilon F, Filosa T, Di Cristofaro L, De Lotto F \& Fallo F. Intima-media thickness measurement of the carotid artery in patients with primary hyperparathyroidism. A prospective case-control study and long-term follow-up. In Vitro $2006 \mathbf{2 0} 887-890$.

38 Rashid G, Bernheim J, Green J \& Benchetrit S. Parathyroid hormone stimulates the endothelial nitric oxide synthase through protein kinase A and C pathways. Nephrology, Dialysis, Transplantation 200722 2831-2837.
39 Nilsson IL, Aberg J, Rastad J \& Lind L. Endothelial vasodilatory dysfunction in primary hyperparathyroidism is reversed after parathyroidectomy. Surgery 1999126 1049-1055.

40 Neunteufl T, Heher S, Prager G, Katzenschlager R, Abela C, Niederle B \& Stefeneli T. Effects of successful parathyroidectomy on altered arterial reactivity in patients with hypercalcaemia: results of a 3-year follow-up study. Clinical Endocrinology 200053 229-233.

41 Kosch M, Hausberg M, Vormbrock K, Kisters K, Gabriels G, Rahn KH \& Barenbrock M. Impaired flow-mediated vasodilation of the brachial artery in patients with primary hyperparathyroidism improves after parathyroidectomy. Cardiovascular Research 2000 47 813-818.

42 Nilsson IL, Rastad J, Johansson K \& Lind L. Endothelial vasodilatory function and blood pressure response to local and systemic hypercalcemia. Surgery 2001130 986-990.

Received 20 February 2010

Accepted 24 February 2010 